\title{
Atmospheric Pressure Chemical Ionization of Alkanes, Alkenes, and Cycloalkanes
}

\author{
S. Ehart Bell, R. G. Ewing, G. A. Eiceman, and Z. Karpas
}

An error in our recently published manuscript has been brought to our attention (J. Am. Soc. Mass Spectrom. 1994, 5, 177-185). On page 182, column 1, we incorrectly cited the proton affinities of cyclohexane and cyclohexene as 189 and $212 \mathrm{kcal} / \mathrm{mol}$ respectively. The correct values are 169 and $189 \mathrm{kcal} / \mathrm{mol}$ (S. G. Lias, J. E. Bartmess, J. F. Liebman, J. L. Holmes, R. D. Levin, and W. G. Mallard, J. Phys, Chem. Ref. Data, Suppl. 1, 1988, 17, 2). The corrected thermodynamic values for reactions 2 and 3 (same page) are therefore:

$$
\begin{aligned}
\mathrm{N}_{2}^{+}+\text {cyclo- } \mathrm{C}_{6} \mathrm{H}_{12} \rightarrow c-\mathrm{C}_{6} \mathrm{H}_{11}^{+}+\mathrm{H}+\mathrm{N}_{2} \\
-103 \mathrm{kcal} / \mathrm{mol} \\
\mathrm{c}_{-} \mathrm{C}_{6} \mathrm{H}_{11}^{+} \rightarrow \mathrm{C}_{6} \mathrm{H}_{9}^{+}+\mathrm{H}_{2} \\
+16 \mathrm{kcal} / \mathrm{mol}
\end{aligned}
$$

The structure of the $\mathrm{C}_{6} \mathrm{H}_{9}^{+}$ion was assumed to be cyclohexene-3-yl. Note also in reaction 2, the $\mathrm{H}$ was incorrectly listed as $\mathrm{H}^{+}$. As a result, this dissociative charge transfer reaction 2 is exothermic with excess energy available to allow further dissociations as in reaction 3. However, dissociative charge transfer from water ions may also lead to hydrogen elimination:

$$
\begin{aligned}
\mathrm{H}_{2} \mathrm{O}^{+}+\mathrm{C}_{-}-\mathrm{C}_{6} \mathrm{H}_{12} \rightarrow & \mathrm{c}-\mathrm{C}_{6} \mathrm{H}_{11}^{+}+\mathrm{H}+\mathrm{H}_{2} \mathrm{O} \\
& -34 \mathrm{kcal} / \mathrm{mol} \\
\mathrm{c}-\mathrm{C}_{6} \mathrm{H}_{11}^{+} \rightarrow & \mathrm{C}_{6} \mathrm{H}_{9}^{+}+\mathrm{H}_{2} \\
& +16 \mathrm{kcal} / \mathrm{mol}
\end{aligned}
$$

Overall, this reaction scheme is exothermic by 18 $\mathrm{kcal} / \mathrm{mol}$. Charge transfer between $\mathrm{H}_{2} \mathrm{O}^{+}$and $c^{-}$
$\mathrm{C}_{6} \mathrm{H}_{12}$, which produces the cyclohexene cation $\left(\Delta \mathrm{H}^{\circ}\right.$ $=-56 \mathrm{kcal} / \mathrm{mol}$ ), also could occur. Thus, hydrogen elimination from cyclohexane possibly could occur after dissociative charge transfer with water ions as well as with nitrogen ions.

Under low moisture conditions in an ion mobility spectrometer, the following reaction possibly could occur to produce $\mathrm{H}_{3} \mathrm{O}^{+}$ions:

$$
\begin{aligned}
\mathrm{H}_{2} \mathrm{O}^{+}+c-\mathrm{C}_{6} \mathrm{H}_{12} \rightarrow & \mathrm{H}_{3} \mathrm{O}^{+}+c-\mathrm{C}_{6} \mathrm{H}_{11} \\
& -45 \mathrm{kcal} / \mathrm{mol}
\end{aligned}
$$

The proton transfer reaction scheme from $\mathrm{H}_{3} \mathrm{O}^{+}$ analogous to reaction 4 is slightly endothermic $(+6$ $\mathrm{kcal} / \mathrm{mol}$ ), but would be favored by a significant change in entropy:

$$
\begin{aligned}
& \mathrm{H}_{3} \mathrm{O}^{+}+\mathrm{c}-\mathrm{C}_{6} \mathrm{H}_{12} \rightarrow \mathrm{c}^{-\mathrm{C}_{6}} \mathrm{H}_{13}^{+}+\mathrm{H}_{2} \mathrm{O} \\
& -2 \mathrm{kcal} / \mathrm{mol} \\
& \mathrm{c}-\mathrm{C}_{6} \mathrm{H}_{13}^{+} \rightarrow \mathrm{c}-\mathrm{C}_{6} \mathrm{H}_{11}^{+}+\mathrm{H}_{2} \\
& +8 \mathrm{kcal} / \mathrm{mol}
\end{aligned}
$$

The relative contributions of the different pathways could not be determined under the given experimental conditions. Our thanks to Dr. John Stone of Queen's University, Kingston, Ontario, for noting these points. 